\title{
A Turing test for artificial intelligence in cancer
}

\author{
The convergence of big data and artificial intelligence is poised to revolutionize cancer research and care, from \\ basic conceptual developments to translational and clinical applications. To reap these benefits, it is important to \\ separate the hope from the hype.
}

T he progressive digitalization of our society, science and technology is transforming cancer research and healthcare. DNA sequencing alone is predicted to surpass other data-intensive fields in terms of amount of information and analytical capacity in the next few years. Electronic health records and the ability to collect health and behavioral data through smartphones and wearable devices add to the wealth of available information. However, the new opportunities to extract, integrate and contextualize larger and more diverse types of datasets about cancer pathobiology, diagnosis and care bring new challenges. Chief among them is the need to develop appropriate storage, filtering and analytical powers, given that handling the staggering amount of available data is beyond the ability of individual researchers and clinicians and is often challenging for research institutes and hospitals. Artificial intelligence (AI) holds the potential to harness big data and to provide new ways to understand, diagnose and treat cancer.

Human beings have long been fascinated with the possibility of automating tasks. Some early references to anthropomorphic automata can be found in Homer's Iliad and, later, in Leonardo da Vinci's descriptions of complex robot designs. More recently, two landmark works paved the way to the modern notion of machine intelligence: in the 19th century, Ada Lovelace proposed the use of the Babbage machine to solve complex problems beyond mere mathematical calculations; and in 1950, Alan Turing presented his test to examine a machine's ability to exhibit intelligent behavior and win a human-imitation game. However, the term 'artificial intelligence' would not be coined until 1956, in the historic Dartmouth Summer Research Project, in which John McCarthy and colleagues proposed some of the founding concepts that defined how this discipline would develop in the ensuing years. Just over six decades later, $\mathrm{AI}$ is employed routinely to enhance procedures such as internet searches, predictive texting, targeted advertising or business optimization.

Cancer research and oncology benefit from machine intelligence and procedural automation through a natural mutualistic convergence. The use of algorithms and statistical modeling to automatize tasks makes the processing of complex datasets fast and efficient. Moreover, AI approaches feed on data and learn while analyzing them, becoming capable of efficient pattern recognition when exposed to new datasets, detecting patterns that would be indiscernible to human perception. Thus, automated pre-filtering and classification systems have a clear impact in exploratory studies aiming to identify basic principles of disease biology and could also benefit patients and doctors by speeding up diagnosis and providing support in reaching better and faster decisions in the clinic. Some successful implementations of AI in cancer research and clinical oncology include the identification of regulatory patterns from proteomics or genomics data; automated cell classification from single-cell technologies; drug screens based on predicted docking on 3D structures; discrimination of benign versus malignant skin lesions from digital pictures; automatic delineation of tumor margins during surgery; prediction of driver mutations, patient survival or drug response from whole digital pathology slides or '-omics' data; and automated breast-cancer screening from mammogram images. Multi-omics data integration in particular provides an excellent subject for the analytical and synthetic abilities of AI. This intensely developing area of cancer research has great potential not only to advance basic cancer biology knowledge but also to improve clinical practice by identifying patients who could benefit from particular therapies, including targeted therapies or immunotherapies. Digital pathology and radiation- and cell-based diagnostics have been profoundly aided by the rapid developments in computer vision and pattern recognition in the past 20 years. The digitalization of electronic health records is enabling the use of natural language processing to find traits that might indicate the earlier stages of cancer. With the advent of the Internet of Things, wearable devices could provide real-time data that could inform on underlying health changes that might reflect early stages of tumor development. These few examples highlight some of the potential applications of machine learning in oncology__ _ it seems that the limit is our imagination.

Similar to any disruptive technology, AI-assisted cancer research and care comes with not only a healthy dose of hype about its potential benefits but also some fears and skepticism about its perceived power. One of the questions that arises is to what extent AI will eventually substitute some of the tasks of trained pathologists and clinicians in diagnosis and treatment decisions. As both humans and machines are prone to err, achieving optimal performance should instead focus on the points where the two converge. For instance, under the supervision of an expert pathologist, the power of AI to classify samples promptly can speed up diagnosis and enhance its accuracy. For analytical abilities that are beyond human capacity, such as the integration in real time of vast amounts of heterogeneous data or forecasting mutations in the absence of sequencing information, AI can provide an additional toolset to support researchers and clinicians. Similar to the diagnostic revolution that X-rays or CT scans represented when they were first widely adopted, new AI-based technologies hold the promise of enhancing clinical performance in hospitals and helping to improve patients' lives.

Nevertheless, the importance of many controlled, proof-of-concept studies of limited size can sometimes be overstated. Even when AI performance is impressive, it is not perfect. Limited sample size and the scarcity of appropriate validation cohorts can result in important caveats, including sample misclassification, detection of non-relevant features in the testing of new datasets, or the lack of generalizability of an algorithm's predictive powers when different settings are used. These shortcomings could be alleviated through exposure to larger, carefully curated datasets that will improve analytical and predictive accuracy and prevent biases. However, privacy concerns about access to and use of personal data cannot be disregarded. Generating biological data and electronic health records of trustable digital identity through robust privacy policies will enable the 
safe and ethical use of multilayered data, including social, behavioral, physical, multi-omics and genealogical data, for the benefit of human health.

An important point to keep in mind when thinking about the impact of AI in cancer is that it must not center only on the few patients who can afford expensive care. It is essential to ensure that the information fed to AI is representative of the broader population, so that results will be widely applicable. Extensive validation in independent cohorts will be critical to achieving this aim. When it comes to routine application, focus should be placed on AI-driven approaches that can enable quick diagnosis at affordable costs with simple implementation. Such efforts could benefit underprivileged parts of the population profoundly, by providing highquality clinical assessment even when access to state-of-the-art medical care is difficult.

To actively engage in this conversation, in this issue of Nature Cancer we present a Viewpoint in which six scientists discuss different uses of AI in cancer research, diagnosis and care. Although this discussion represents a subset of expertise and potential applications in a vast field, we hope that our readers will find their thoughts on recent developments, challenges and future directions stimulating.

The cooperation of humans and AI has the potential to enhance cancer research, improve clinical practice and alleviate human suffering in a unique manner. Going back to Alan Turing's test from 70 years ago, the goal here is not to see if machines can think, but rather if they can assist us, beyond our current abilities, to fight cancer in an intelligent and panhuman manner.

Published online: 24 February 2020

https://doi.org/10.1038/s43018-020-0041-7 